Artículo Original

\title{
Descripción de un nuevo género y especie de Salpinginae (Coleoptera: Salpingidae) para Chile
}

Description of a new genus and species of Salpingidae (Coleoptera: Salpingidae) from Chile

Jaime Solervicens ${ }^{1}$

${ }^{1}$ Sociedad Chilena de Entomología, Santiago, Chile. E-mail: jaime.solervicens@umce.cl

\section{ZooBank: urn:lsid:zoobank.org:pub:1A078329-9918-4ACD-A040-41C56C855D9C https: / / doi.org/10.35249/ rche.47.1.21.02}

Resumen. Basado en material recolectado en la cordillera de Nahuelbuta (provincias de Malleco y Arauco) se describe un nuevo género y especie de Salpingidae de Chile. Se presentan imágenes del adulto de la especie y de detalles de la cabeza y el tórax, además de figuras de las piezas bucales, alas y órganos genitales del macho y de la hembra. Se destacan sus caracteres diagnósticos y se establecen diferencias y afinidad con otros géneros de la familia.

Palabras clave: Provincia del Maule; Región Andina; taxonomía.

Abstract. Based on material collected in the Nahuelbuta mountain range (Malleco and Arauco provinces) a new genus and species of Salpingidae from Chile are described. Images of the adult of the species and structural details of the head and thorax are presented, as well as figures of the mouthparts, wings, and male and female genitalia. Its diagnostic characteristics are highlighted and its differences and affinity with other genera of the family are established.

Key words: Andean Region; Maule province; taxonomy.

\section{Introducción}

Un grupo de salpinginos indeterminados provenientes de las regiones de La Araucanía y Biobío no pudo ser clasificado en ninguno de los géneros conocidos para Chile (Elgueta y Arriagada 1989; Solervicens 2020). Tampoco pudo ser reconocido como uno de los géneros citados para otras regiones del mundo (Blair 1919; Blackwelder 1945; Spilman 1954; Lawrence y Britton 1991; Pollock 2002; Pollock y Löbl 2008). Sobre esta base se estima que los coleópteros citados inicialmente corresponden a nuevos taxones del nivel género y especie, los que se describen en esta contribución.

\section{Materiales y Métodos}

El material en que se basa este estudio corresponde a 60 ejemplares provenientes de la cordillera de Nahuelbuta. Las medidas del cuerpo se tomaron mediante un estereomicroscopio provisto de reglilla graduada; ya sea que se trate de un valor promedio o de relaciones de medidas siempre se indica su rango. Para efecto de la disección de

Recibido 8 Noviembre 2020 / Aceptado 11 Enero 2021 / Publicado online 26 Febrero 2021 Editor Responsable: José Mondaca E. 
estructuras se trató los ejemplares en una solución caliente de hidróxido de potasio; las piezas disectadas se conservaron junto al insecto. El largo del cuerpo se consideró entre el borde anterior de la cabeza y el ápice de los élitros. Las fotografías se tomaron con una cámara digital Panasonic LX3 a través del ocular de un estereomicroscopio Olympus SZ51. Los esquemas lineales se efectuaron a partir de fotografías mediante el programa de dibujo SketchBook, complementados por la observación bajo microscopio. La diferenciación del nuevo género respecto de los demás representantes de la subfamilia se basó en las descripciones disponibles (Champion 1889, 1916; Pic 1903, 1919; Blair 1919; Lea 1919; Iablokoff-Khnzorian 1985; Anderson y Fuller 2005; Alekseev 2017), claves de determinación (Blair 1919; Iablokoff-Khnzorian 1985; Pollock 2002) e imágenes y comprende básicamente caracteres de la morfología externa. La terminología empleada en las descripciones sigue a la propuesta por Lawrence et al. (2010). El material estudiado queda depositado en la colección del Museo Nacional de Historia Natural de Chile, Santiago, Chile (MNNC), en la del Instituto de Entomología de la Universidad Metropolitana de Ciencias de la Educación, Santiago, Chile (IEUMCE) y en la colección particular de Víctor Manuel Diéguez, Santiago, Chile.

\section{Resultados}

\section{Nawelbuta gen. nov.}

(Figs. 1-13)

\section{Especie tipo: Nawelbuta foveognathus sp. nov.}

Diagnosis. Cuerpo de alrededor de 4 milímetros de largo y poco más de tres veces más largo que ancho; cabeza no prolongada en rostro, ancha; pronoto con su mayor amplitud en parte anterior, apenas más ancho que la cabeza; élitros de lados subparalelos. Puntuación general a base de puntos redondos, en los élitros dispuesta en filas o desordenada. Pilosidad a base de pelos finos semitendidos y pelos largos, erectos, intercalados (Fig. 1).

Descripción. Cabeza (Figs. 1, 2): orientada oblicuamente hacia abajo, más ancha que larga; rebordes antenales bien marcados, convergentes hacia adelante, alcanzan la concavidad lateral del clípeo; sutura frontoclipeal no marcada, insinuada a los lados por depresión; clípeo en ambos extremos con bordes cóncavos que forman un conducto hacia la base de las mandíbulas (Fig. 8a); temples cortos. Labro transverso, borde anterior recto. Ojos laterales redondeados, sobresalientes, enteros, con pelos cortos entre los omatidios, éstos pequeños. Antenas (Fig. 5) sobrepasan los ángulos humerales, segmentos gradual y moderadamente ensanchados hacia el ápice. Mandíbulas (Figs. $8,8 a)$ bidentadas, con fóvea basal en superficie dorsal junto a la articulación anterior, margen mesal provisto de un peine en parte media y entre este y la mola una corta prosteca, mola plana, amplia. Maxilas (Fig. 9): galea ancha, rectangular, lacinia angosta, ambas con mechón apical de cerdas, ápice interno de lacinia con uncus, palpos sobresalen al costado de las mandíbulas, segundo segmento cónico fuertemente angostado hacia la base, tercer segmento cónico, poco más corto que el segundo y con base más ancha, cuarto dos veces más largo que el tercero, con ápice truncado. Labio (Fig. 10): mentón corto y fuertemente transverso, redondeado en los extremos, prementón cuadrangular algo ensanchado hacia el ápice, homogéneamente esclerosado, borde distal subrecto y ángulos laterales redondeados, palpos labiales pequeños, no sobresalientes, último segmento ovoide, angostado hacia el ápice. Gula (Fig. 2) ancha, trapezoidal, levemente más angosta hacia adelante, convexa, sin puntos, suturas gulares rectas, impresiones tentoriales bien marcadas. Submentón tan largo como la gula, su parte distal curvada hacia ventral. Áreas paragulares convexas, separadas del resto de la superficie ventral por 
una depresión transversa que alcanza la zona posterior del ojo. Tórax: pronoto (Figs. 1, 3): levemente más ancho que la cabeza y ligeramente más largo que ancho, ancho anterior un poco mayor que el posterior; máxima amplitud en tercio anterior; costados sin reborde lateral, redondeados, en vista dorsal rectos y divergentes en dos tercios basales y curvos en el distal; borde anterior recto, finamente carenado, borde posterior con dos carenas finas subparalelas divergentes al costado; disco convexo transversalmente y subrecto longitudinalmente, depresiones prebasales laterales presentes; hipómero amplio; proceso postcoxal corto; sutura notoesternal marcada; prosternón trapezoidal, convexo, amplio, dos veces el largo antero-posterior de una coxa, proceso prosternal linear, ligeramente ensanchado en el ápice; cavidades coxales anteriores redondas, abiertas, contiguas (Fig. 3). Mesoventrito (Fig. 4) tan largo como el prosternón, triangular, convexo, proceso mesoventral largo; suturas mesanepisternales marcadas, mesanepisternos contactados en la línea media anterior; cavidades coxales medias redondas, cerradas. Metaventrito (Fig. 4) aproximadamente 1,5 veces el largo del mesoventrito, convexo transversalmente; proceso metaventral corto; superficie con depresión longitudinal media posterior; sutura metakatepisternal marcada; espacio intercoxal posterior subrecto. Escutelo redondeado. Élitros poco más de dos veces más largos que su ancho basal y poco menos de tres veces más largos que el pronoto; ángulos humerales marcados; costados subrectos, ligeramente más anchos en la mitad que en la base, regularmente redondeados en tercio distal; disco transversalmente convexo, declive distal breve, oblicuo. Epipleura ancha bajo el ángulo humeral, gradualmente angostada hacia atrás, termina a nivel del segundo ventrito. Alas metatorácicas (Fig. 7) bien desarrolladas, R1+2 engrosada distalmente; celda radial mal delimitada basalmente, invadida por el ensanchamiento de R1+2; r3 débilmente marcada, larga; 4 ausente; RP poco más larga que el espolón de MP1+2; MP3+4 unida o separada de MP1+2, rama MP3 ausente; celda en cuña presente; AA4 ausente; AP3+4 presente. Patas: coxas anteriores cónicas, sobresalientes, coxas medias redondas; trocánteres pequeños, su unión con el fémur oblicua; fémures fusiformes, fuertemente angostados hacia la base; tibias moderadamente ensanchadas hacia el ápice, subrectas, con peines de cerdas distales y dos espolones apicales; tarsos (Fig. 6) 5-5-4, segmentos 2 y 3 de patas anteriores y medias y segmento 2 de patas posteriores fuertemente escotados por dorsal en el ápice y ensanchados por ventral en un lóbulo triangular de borde distal entero; penúltimo segmento de todos los tarsos pequeño, cilíndrico, contenido en la escotadura del precedente; segmentos 1 a 3 de tarsos anteriores y medios y 1 y 2 de los posteriores con cepillo de pelos ventral; garras pretarsales simples. Abdomen: con cinco ventritos de tamaño decreciente del 1 al 5, convexos transversalmente, 1 a 4 con borde posterior recto, 5 con borde redondeado, primer ventrito con proceso intercoxal agudo. Pigidio con borde posterior redondeado, superficie convexa. Macho: octavo esternito poco esclerosado, con borde distal levemente escotado al centro, sin apodema; noveno esternito con spiculum gastrale largo. Edeago (Figs. 11, 12): parámeros completamente fusionados formando el lóbulo apical, que se articula con la falobase; lóbulo apical de forma cónica y con apéndices accesorios, en su parte ventral anterior se asocia a dos escleritos; falobase sin apodemas; pene cilíndrico, corto, con apodemas. Hembra: octavo esternito poco esclerosado, con largo spiculum ventrale. Ovipositor (Fig. 13) largo, membranoso, cerca de seis veces más largo que ancho, báculos ventrales y dorsales largos, de longitud similar, los últimos más finos que los primeros, báculos oblicuos finos; lámina ventral con esclerosamiento central entre los coxitos; lámina dorsal con superficie estriada y ápice piloso; coxitos alargados, con cuatro divisiones, estilos bien desarrollados.

Etimología. El nombre del género, en lengua mapudungun, se establece de acuerdo a la localidad de recolección de los ejemplares de su especie tipo. 


\section{Nawelbuta foveognathus sp. nov.}

(Figs. 1-13)

= Genus sp.; Arias et al. 2008 (Appendix S1)

Descripción. Cuerpo de 4,24 milímetros de largo promedio (rango: 3,8-4,8, n=10), 3,44 veces más largo que ancho (rango; 3,3-3,5, n=7), cabeza ancha, pronoto cordiforme, élitros de lados subparalelos. Coloración: cabeza, incluidas antenas y piezas bucales, y protórax anaranjados a rojizos, a veces cabeza o protórax más oscuros, ápice de mandíbulas negro; élitros testáceo claro a pardo o pardo rojizo, meso y metatórax pardo rojizo oscuro; abdomen pardo a pardo rojizo; patas testáceas. Puntuación densa y homogénea, puntos separados mayoritariamente por el diámetro de un punto, en el pronoto se insinúa a veces una estrecha franja media longitudinal sin puntos; en los élitros los puntos pueden distribuirse en líneas junto a interestrías puntuadas o la puntuación estar desorganizada, las líneas, cuando presentes, mal conformadas al costado después de la séptima, la puntuación en general borrada o reducida en el ápice y junto a la sutura en el tercio distal; abdomen con puntos de menor tamaño. Pilosidad de cabeza y pronoto con pelos de largo mediano, curvos, inclinados, blanquecinos, acompañados de pelos largos, rectos, erguidos y ralos; élitros con pelos rectos o subrectos, inclinados hacia atrás, junto a pelos largos, rectos o poco curvados, ralos y erguidos; partes ventrales con pelos cortos, tendidos, junto a escasos pelos largos, erguidos (Fig. 1). Cabeza (Figs. 1, 2): 1,33 veces más ancha que larga (rango: 1,22-1,42, n=10) y casi tan ancha como la mayor amplitud del pronoto (relación promedio 0,94; rango: 0,92$1,00, n=10)$; frente convexa, generalmente la parte anterior con dos depresiones laterales que alcanzan el borde basal del clípeo; reborde antenal sobresaliente, subrecto, más alto en parte media, con margen superior agudo; clípeo sobresaliente; labro convexo, costados redondeados; temples menores que el diámetro antero posterior del ojo; antenas (Fig. 5) sobrepasan ligeramente los ángulos humerales, 1,47 veces más largas que el pronoto (rango: 1,43-1,59, n=8), escapo oblongo, pedicelo poco más de la mitad del largo del escapo, tercer segmento largo como el escapo, cuarto a décimo de largo similar, algo más cortos que el tercero y gradualmente más anchos, 7 a 10 triangulares, 11 casi dos veces más largo que el precedente, fusiforme. Tórax: pronoto (Figs. 1,3): su largo ligeramente mayor o igual que el ancho máximo (relación promedio 1,03, rango: 1,00-1,11, n=10), ancho anterior ligeramente mayor que el posterior (promedio 1,09, rango: 1,06-1,15), depresiones prebasales laterales leves, puntuadas; hipómero liso, sin puntos; prosternón con franja anterior sin puntos y arrugamiento transverso. Proceso mesoventral angosto, aproximadamente un cuarto del ancho de la coxa adyacente (Fig. 4). Depresión longitudinal del metaventrito oblonga (Fig. 4), ubicada en mitad posterior, el área circundante aplanada, con puntuación menor y más espaciada; sutura metakatepisternal deprimida y puntuada, el área anterior adyacente algo abombada, lisa y sin puntos. Escutelo puntuado, algo aguzado en el ápice. Élitros 2,21 veces más largos que el ancho humeral (rango: 2,01-2,32, n=10) y 2,8 veces más largos que el pronoto (rango: 2,70-2,96, $\mathrm{n}=10$ ), el ancho humeral es 1,27 veces el ancho máximo del pronoto (rango: 1,21-1,35, n=10), la mayor amplitud de la parte media es 1,09 veces la humeral (rango: 1,06-1,16, $\mathrm{n}=10$ ). Abdomen: ventrito 5 con leve curvatura hacia ventral en ambos sexos. Noveno esternito de machos con spiculum gastrale largo, alrededor de dos veces la longitud de los brazos. Edeago (Figs. 11, 12) con el extremo del lóbulo apical ligeramente curvado hacia ventral y apéndices accesorios finos, provistos de larga cerda distal. Ovipositor (Fig. 13) con lámina ventral provista de esclerosamiento angosto, aguzado hacia la base.

Etimología. El nombre de la especie se refiere a la presencia de una fóvea en la base de la mandíbula. 

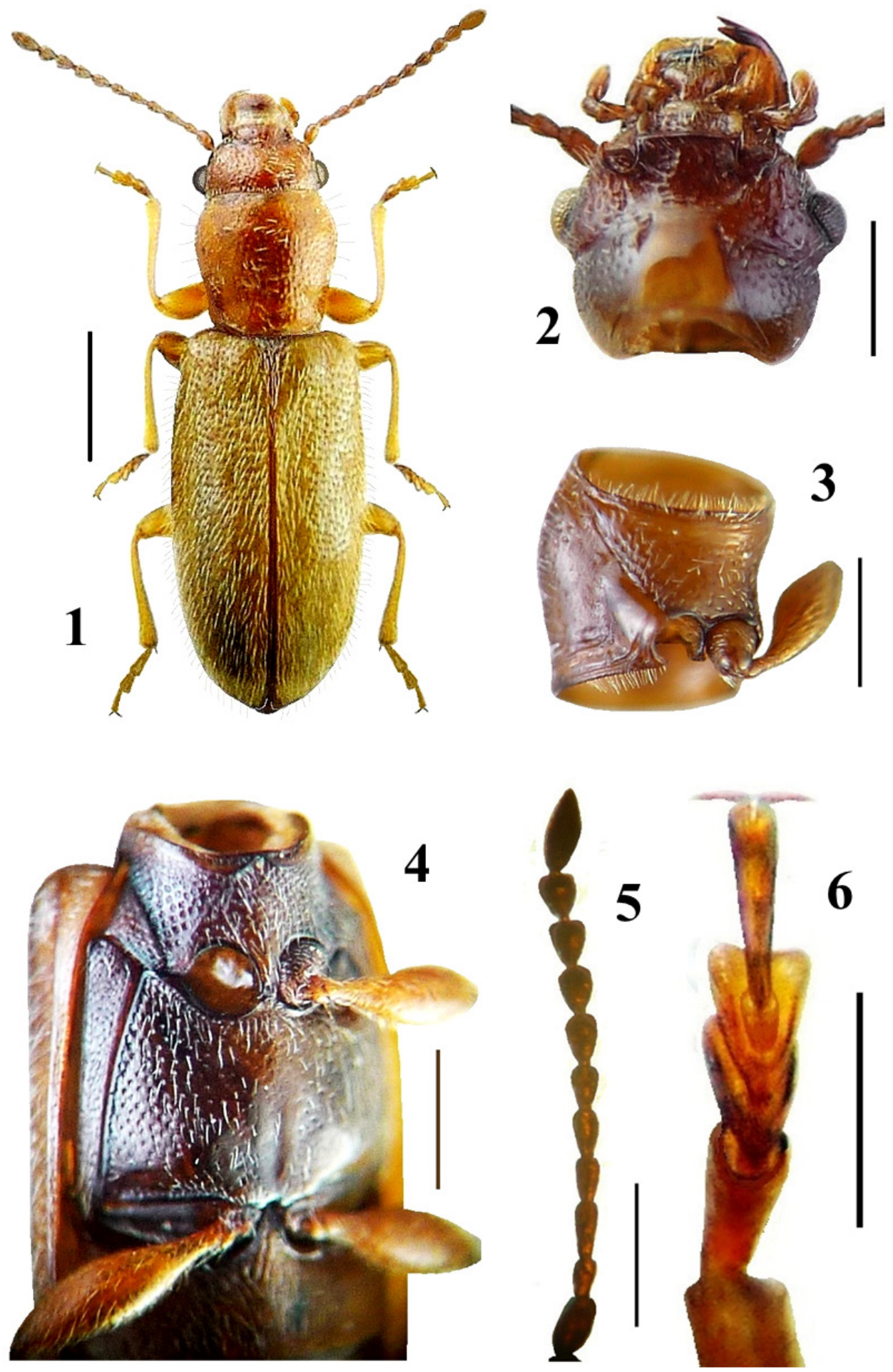

Figuras 1-6. Nawelbuta foveognathus. 1. Hábito dorsal. Escala: $1 \mathrm{~mm}$. 2. Cabeza, vista ventral. Escala: 0,5 $\mathrm{mm}$. 3. Protórax, vista lateroventral. Escala: 0,5 mm. 4. Meso y metatórax, vista lateroventral. Escala: 0,5 mm. 5. Antena. Escala: 0,25 mm. 6. Protarso. Escala: 0,25 mm. / 1. Dorsal habitus. Scale: $1 \mathrm{~mm}$. 2. Head, ventral view. Scale: $0.5 \mathrm{~mm}$. 3. Prothorax, lateroventral view. Scale: $0.5 \mathrm{~mm}$. 4 . Meso and metathorax, lateroventral view. Scale: $0.5 \mathrm{~mm}$. 5. Antenna. Scale: $0.25 \mathrm{~mm}$. 6. Protarsus. Scale: $0.25 \mathrm{~mm}$. 

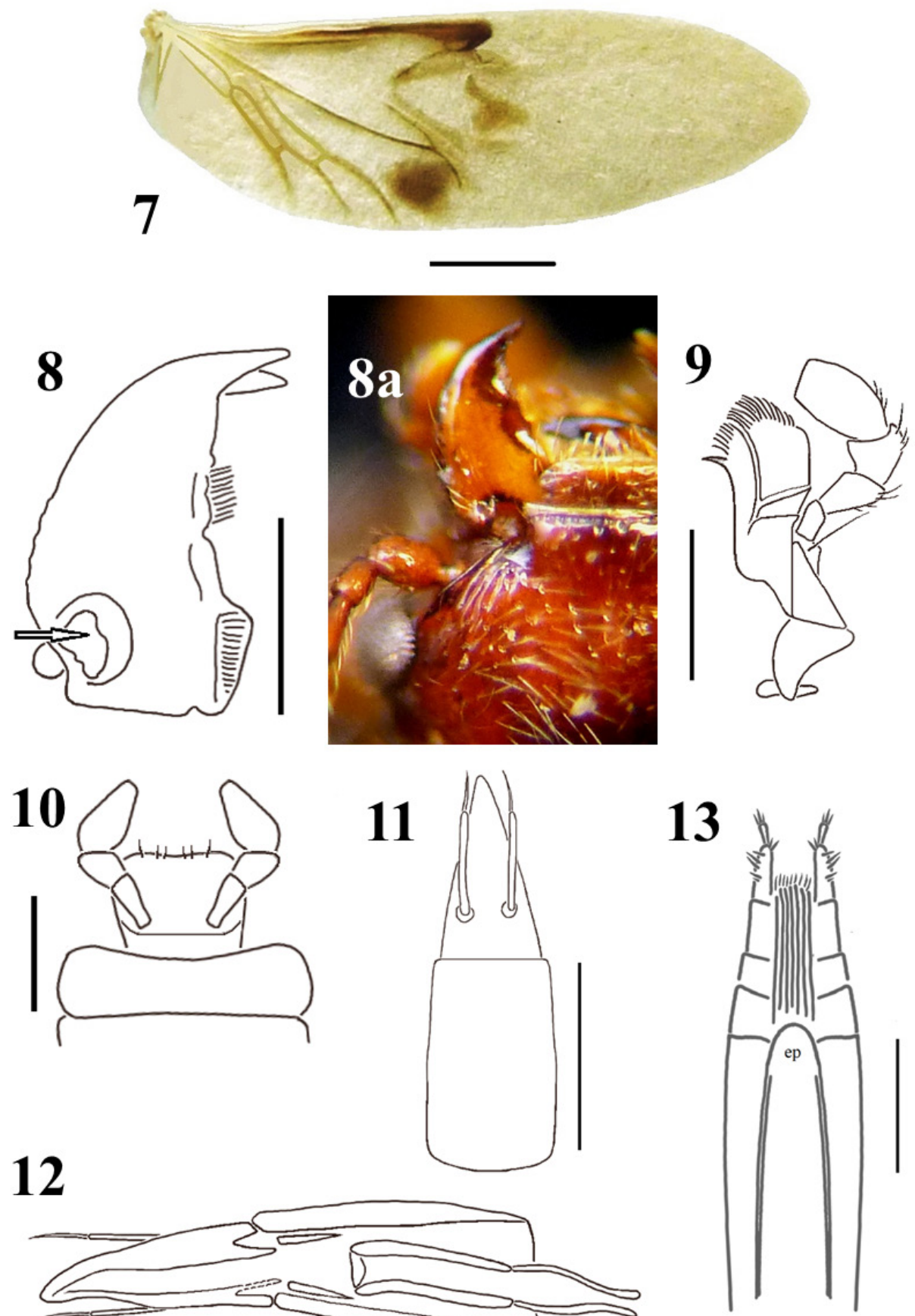

Figuras 7-13. Nawelbuta foveognathus. 7. Ala metatorácica. Escala: $1 \mathrm{~mm}$. 8. Mandíbula izquierda, vista dorsal (flecha indica fosa mandibular). Escala: $0,22 \mathrm{~mm}$. 8a. Fóvea basal de la mandíbula y conducto formado por la concavidad lateral del clípeo. 9. Maxila. Escala: 0,20 mm. 10. Labio. Escala: 0,15 mm. 11-12. Edeago, vistas dorsal y lateroventral. Escala: $0,25 \mathrm{~mm}$. 13. Ovipositor, parte distal, vista dorsal. Escala: 0,25 mm. / 7. Metathoracic wing. Scale: $1 \mathrm{~mm}$. 8. Left mandible, dorsal view (arrow indicates mandibular fossa). Scale: $0.22 \mathrm{~mm}$. 8a. Basal fovea of the mandible and canal formed by the lateral concavity of the clypeus. 9. Maxilla. Scale: $0.20 \mathrm{~mm}$. 10. Labium. Scale: $0.15 \mathrm{~mm}$. 11-12. Aedeagus, dorsal and lateroventral views. Scale: $0.25 \mathrm{~mm}$. 13. Ovipositor, distal part, dorsal view. Scale: $0.25 \mathrm{~mm}$. 
Material tipo. Holotipo: 1 macho, 11-CHILE, VIII Región, P N Nahuelbuta, Pichinahuel Exit, 3748.316’ S/07302.086’W/1182 m, 07.XII.2001, Canopy Fogging GT, Araucaria araucana Male, Arias et al. UC Berkeley. Paratipos: 1 macho, 10-CHILE, VIII Región, P

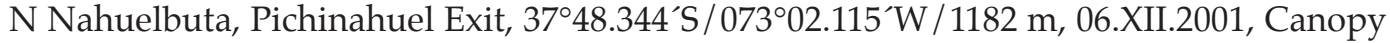
Fogging GT, Araucaria araucana Female \& Male, Arias et al. UC Berkeley; 1 macho, 9-CHILE, VIII Región, P N Nahuelbuta, Pichinahuel Exit, 3748.284`S /07302.112 'W/ 1182 m, 06.XII.2001, Canopy Fogging GT, Araucaria araucana Male \& Fem, Arias et al. UC Berkeley; 1 hembra, 10-CHILE, VIII Región, P N Nahuelbuta, Pichinahuel Exit,

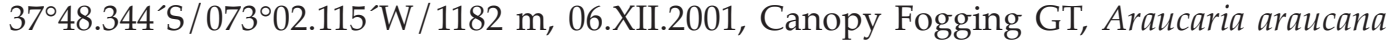
Female \& Male, Arias et al. UC Berkeley; 1 ejemplar sexo no determinado, 5-CHILE, IX Región, Nahuelbuta (Out side), 1 VIII 2003, 3748.30`S/07302.0`W, Fogging/Araucaria araucana, Arias et al. UC Berkeley; 4 ejemplares sexo no determinado, 9-CHILE, VIII Región, P N Nahuelbuta, Pichinahuel Exit, 3748.284’S /07302.112`W/ 1182 m, 06.XII.2001,Canopy Fogging GT, Araucaria araucana Male \& Fem, Arias et al. UC Berkeley; 1 ejemplar sexo no determinado, 12-CHILE, VIII Región, P N Nahuelbuta, Pichinahuel Exit, 3748.386’S $/ 073^{\circ} 02.180^{\prime} \mathrm{W} / 1181$ m, 07.XII.2001,Canopy Fogging GT, Araucaria araucana Female, Arias et al. UC Berkeley; 2 ejemplares sexo no determinado, montados sobre el mismo alfiler, Arauco, Caramávida, 23-XII-1953, Col. L. Peña (holotipo y 11 paratipos especificados anteriormente depositados en MNNC); 1 macho abdomen disectado, ChileMalleco, Nahuelbuta, 5-II-80, J. Solervicens; 1 macho abdomen y ala disectados, ChileMalleco, Nahuelbuta, 7-II-80, J. Solervicens; 3 hembras, Chile, Malleco, P.N. Nahuelbuta, 13/02/1997, J. Solervicens (una hembra con abdomen disectado); 1 hembra abdomen y ala disectados, Chile, Malleco, Nahuelbuta, 10/3/1999, P. Estrada; 4 ejemplares sexo no determinado, Chile, Malleco, P.N. Nahuelbuta, 13/02/1997, J. Solervicens (depositados en IEUMCE).

Material adicional estudiado. 5 ejemplares, 9-CHILE, VIII Región, P N Nahuelbuta, Pichinahuel Exit, 3748.284’S /07302.112`W / 1182 m, 06.XII.2001, Canopy Fogging GT, Araucaria araucana Male \& Fem, Arias et al. UC Berkeley (MNNC); 2 ejemplares, 10-CHILE, VIII Región, P N Nahuelbuta, Pichinahuel Exit, 3748.344’S/07302.115`W/1182 m, 06.XII.2001, Canopy Fogging GT, Araucaria araucana Female \& Male, Arias et al. UC Berkeley (MNNC). 3 ejemplares Chile, IX Región, P.N. Nahuelbuta, 25 / 30 enero 2005, Leg. V.M. Diéguez, en Araucaria araucana (en colección V.M. Diéguez); 3 machos, Chile, Malleco, P.N. Nahuelbuta, 13/02/1997, J. Solervicens; 1 macho, Chile-Malleco, Nahuelbuta, 5-II80, J. Solervicens; 3 hembras, Chile-Malleco, Nahuelbuta, 4, 5 y 7-II-80, J. Solervicens; 4 hembras, Chile, Malleco, P.N. Nahuelbuta, 13/02/1997, J. Solervicens; 5 hembras Chile, Malleco, Nahuelbuta, 10 y 11/3/1999, 2 P. Estrada, 3 J. Solervicens; 4 ejemplares sexo no determinado, Chile-Malleco, Nahuelbuta, 4, 5 y 7-II-80, J. Solervicens; 8 ejemplares sexo no determinado, Chile, Malleco, Nahuelbuta, 10 /3/1999, 3 P. Estrada, 5 J. Solervicens (IEUMCE).

Hábitat y distribución. Los ejemplares fueron obtenidos en la zona del Bosque AltoMontano de Nahuelbuta (Gajardo 1994); para 20 de ellos hay información de haberse recolectado en Araucaria araucana (Molina) K. Koch, 3 por sacudido de follaje y 17 mediante fogging, en tanto que los 40 restantes fueron obtenidos presumiblemente por sacudido de follaje, pero sin indicación de planta hospedante. Los registros de recolección indican la presencia regular de adultos entre diciembre y marzo, habiéndose recolectado un ejemplar en agosto. En cuanto a su distribución geográfica, los registros actuales señalan su presencia sólo en la cordillera de Nahuelbuta, tanto en el parque nacional (58 ejemplares, 16 de ellos en sector Pichinahuel), como en Caramávida (2 ejemplares), lo que sugiere un posible carácter endémico en el sector. 
Discusión. Nawelbuta gen. nov. se distingue de un grupo numeroso de géneros de Salpinginae que tienen maza antenal de tres segmentos y/o denticulaciones setíferas a los costados del pronoto, tales como Chilopeltis, Istrisia, Lanthanus, Lissodema, Neosalpingus, Notosalpingus, Platamops, Platysalpingus, Salpidema, Sosthenes, Tasmosalpingus y Trichosphaeriestes, por presentar antenas gradualmente ensanchadas hacia el ápice y costados del pronoto redondeados, sin denticulaciones. Otros géneros se diferencian del nuevo taxón por presentar un rostro largo (Cariderus, Salpingus), o patas posteriores con fémures dilatados y tibias con un proceso alargado (Oncosalpingus). Una conformación similar de las antenas junto a costados del pronoto inermes y un rostro corto es compartido por Nawelbuta con Colposis, Poophylax, Pseudorabocerus, Rabocerus, Sphaeriestes y Vincenzellus. Nawelbuta se separa de estos géneros, principalmente, por la organización de sus tarsos, con segmentos 2 y 3 de patas anteriores y medias y segmento 2 de patas posteriores ensanchados hacia el ápice y profundamente escotados por dorsal y penúltimo segmento muy pequeño, cilíndrico, e insertado en la escotadura del precedente, dando una condición pseudotetrámera en los tarsos anteriores y medios (Fig. 6) y pseudotrímera en los posteriores. El único género con una condición similar de los tarsos es Poophylax, con dos especies de las Islas Falkland y la Patagonia Argentina (Anderson y Fuller 2005); sin embargo, los tarsos de estas dos especies tienen los tres primeros segmentos (dos primeros segmentos en patas posteriores) dilatados y escotados y el penúltimo, si bien pequeño y alojado en la escotadura del precedente, sobresale un poco de este y está ensanchado distalmente, marcando con ello cierta diferenciación con respecto a los tarsos de Nawelbuta. Por otra parte, una especie de Sphaeriestes, S. aeratus Mulsant, 1859, muestra una organización de los tarsos similar a la que tiene Poophylax, pero se presenta sólo en patas anteriores de machos, lo cual está posiblemente relacionado con la cópula.

Si la conformación de los tarsos puede relacionar Poophylax y Nawelbuta otros caracteres establecen diferencias significativas. Una relación de las diferencias entre ambos géneros se indica en la tabla 1.

Tabla 1. Diferencias morfológicas entre los géneros Nawelbuta y Poophylax. / Morphological differences between the genera Nawelbuta and Poophylax.

\begin{tabular}{|l|l|l|}
\hline Caracteres & Nawelbuta & Poophylax \\
\hline Dimorfismo sexual & ausente & presente \\
\hline Pilosidad & notoria & Poco notoria \\
\hline Borde anterior labro & recto & escotado \\
\hline Fosa dorsal de la mandíbula & presente & ausente \\
\hline Mola de la mandíbula & presente & ausente \\
\hline Estrías elitrales & mal definidas o ausentes & bien definidas \\
\hline Parámeros del edeago & más cortos que falobase & más largos que falobase \\
\hline Parámeros del edeago & cónicos & rectos \\
\hline Parámeros del edeago & con apéndices accesorios & sin apéndices accesorios \\
\hline Falobase del edeago & recta & globosa \\
\hline Alas posteriores & presentes & ausentes \\
\hline
\end{tabular}

Sobre la base de la conformación de los tarsos se podría postular que Nawelbuta y Poophylax podrían haber tenido un ancestro común presente en la parte austral de Sudamérica y que procesos vicariantes hayan aislado las poblaciones originales al oriente (Poophylax) y poniente (Nawelbuta) del territorio ancestral. Es importante destacar que 
Nawelbuta se encuentra, de acuerdo con los registros actuales, restringida en su distribución geográfica a la cordillera de Nahuelbuta que, así como sectores cordilleranos adyacentes a Nahuelbuta, son considerados como zona de refugio biótico durante los períodos glaciales pleistocénicos (Villagrán y Armesto 2005; Villagrán e Hinojosa 2005; Smith-Ramírez et al 2019). Por otro lado, el nivel de diferenciación entre ambos géneros, que se observa en los caracteres de la tabla 1, en especial lo relativo a la fosa de la mandíbula, mola mandibular y conformación del edeago, constituyen diferencias marcadas a considerar en la posible relación de parentesco entre estos taxones.

En este sentido, la presencia en Nawelbuta de una fóvea mandibular comunicada al exterior por un conducto ubicado en los extremos del clípeo, a lo cual parece asociarse el gran desarrollo de los rebordes antenales que confluyen hacia dicho conducto, es un órgano muy especial, de función desconocida, que, aparentemente, no tiene antecedente entre los géneros de Salpinginae.

\section{Agradecimientos}

A Mario Elgueta D. curador de la colección entomológica del Museo Nacional de Historia Natural de Santiago, Chile, por la obtención de referencias, por facilitar parte de los ejemplares objeto de esta contribución y por la revisión del manuscrito. A Manuel Diéguez por el préstamo de ejemplares. Otros ejemplares estudiados fueron recolectados gracias al proyecto DEB 445413 de la National Science Foundation, otorgado a E.T. Arias \& K.W. Will. Especial reconocimiento a Jiri Hájek, Departamento de Entomología, Museo de Historia Natural de Praga, República Checa, por el préstamo de ejemplares de diversos géneros de Salpingidae del hemisferio norte.

\section{Literatura Citada}

Alekseev, V.I. (2017) Third species of Salpinginae (Coleoptera: Tenebrionoidea: Salpingidae) from Baltic amber. Palaeodiversity, 10: 117-121.

Anderson, R. y Fuller, J. (2005) Poophylax villosa, a new species of Salpinginae (Coleoptera: Salpingidae) from the Falkland Islands. The Coleopterists Bulletin, 59(4): 423-432.

Arias, E.T., Richardson, B.J. y Elgueta, M. (2008) The canopy beetle faunas of Gondwanan element trees in Chilean temperate rain forests. Journal of Biogeography, 35(5): 914-925. Appendix S1.

Blackwelder, R.E. (1945) Checklist of the Coleopterous Insects of Mexico, Central America, the West Indies, and South America. Bulletin United States National Museum, 185(3): 343550.

Blair, K.G. (1919) Notes on the Pythidae with descriptions of new species. Entomologist's Monthly Magazine, 55: 112-124.

Champion, Ch. (1889) Pythidae. En: Biologia Centrali-Americana, vol 4, part 2, pp 1-120.

Champion, Ch. (1916) A new genus of Pythidae from the Falkland Islands. The Annals and Magazine of Natural History, 17(8): 311-313.

Elgueta, M. y Arriagada, G. (1989) Estado actual del conocimiento de los coleópteros de Chile (Insecta: Coleoptera). Revista Chilena de Entomología, 17: 5-60.

Gajardo, R. (1994) La vegetación natural de Chile. Clasificación y distribución geográfica. Editorial Universitaria, Santiago, Chile, 165 pp.

Iablokoff-Khnzorian, S.M. (1985) Les Pythidae paléartiques (Coleoptera). Deutsche Entomologische Zeitschrift, 32(1-3): 193-229.

Lawrence, J.F. y Britton, E.B. (1991) Coleoptera (Beetles). Pp. 543-683. En: Csiro Division of Entomology (ed.) The Insects of Australia: A Textbook for Students and Research Workers, Second Edition. Vol 2. Melbourne University Press, Carlton, 1137 pp. 
Lawrence, J.F., Beutel, R.G., Leschen, R.A.B. y Ślipiński, A. (2010) Glossary of morphological terms, pp. 9-20. En: Leschen, R.A.B., Beutel, R.G. \& Lawrence, J.F. (eds.), Handbook of Zoology, Coleoptera, Beetles, Vol. 2: Morphology and Systematics (Elateroidea, Bostrichiformia, Cucujiformia). Walter de Gruyter GmbH \& Co., Berlín, New York, 785 pp.

Lea, A.M. (1919) New species of Australian Coleoptera. Pythidae. Proceedings of the Linnean Society of New South Wales, 43: 743 [1918].

Pic, M. (1903) Contribution a l'étude du genre Salpingus Gylh. L’Echange, 19: 140.

Pic, M. (1919) Nouvetés diverses. Mélanges Exotico- Entomologiques, 30: 1-21.

Pollock, D.A. (2002) 116 Salpingidae Leach, 1815. Pp. 544-548. En: Arnett, R. H. Jr., Thomas, M.C. Skelley, P.E. y Frank, J. H. (eds.) American Beetles, Volumen 2. Polyphaga: Scarabaeoidea through Curculionoidea. CRC Press, Gainesville, 861 pp.

Pollock, D.A. y Löbl, I. (2008) Family Salpingidae Leach,1815, pp. 417-421. En: Löbl, I. y Smetana, A. (eds.) Catalogue of Palaearctic Coleoptera, vol. 5. Tenebrionoidea. Apollo Books Stenstrup, 670 pp.

Smith-Ramírez, C., Teillier, S., Jiménez, J. E., Barahona-Segovia, R.M., Parra, L.E., Vera, A. y Jerez, V. (2019) Plantas y animales endémicos de la Cordillera de la Costa de Chile. Pp. 393-416. En: Smith-Ramírez, C. y Squeo, F.A. (eds.) Biodiversidad y Ecología de los Bosques Costeros de Chile. Editorial Universidad de Los Lagos, Osorno, Chile. 640 pp.

Solervicens, J. (2020) Rhinolissomus, nuevo género para Rhinosimus valdivianus Philippi y Philippi (Coleoptera: Salpingidae). Revista Chilena de Entomología, 46(3): 373-379.

Spilman, T.J. (1954) Generic names of the Salpingidae and their type species (Coleoptera). Journal of the Washington Academy of Science, 44: 85-94.

Villagrán, C. y Armesto, J.J. (2005) Fitogeografía histórica de la Cordillera de la Costa de Chile, pp. 99-116. En: Smith-Ramírez, C., Armesto, J.J. y Valdovinos, C. (eds.) Historia, Biodiversidad y Ecología de los bosques costeros de Chile. Editorial Universitaria, Santiago. 708 pp.

Villagrán, C. y Hinojosa, L.F. (2005) Esquema biogeográfico de Chile. Capítulo 33: pp. 551-577. En: Llorente Bousquets, J. y Morrone, J.J. (eds.) Regionalización Biogeográfica en Iberoámeríca y tópicos afines. Ediciones de la Universidad Nacional Autónoma de México, Jiménez Editores, México, 577 pp.

Bosques Costeros de Chile. Editorial Universidad de Los Lagos, Osorno, Chile. 640 pp.

Solervicens, J. (2020) Rhinolissomus, nuevo género para Rhinosimus valdivianus Philippi y Philippi (Coleoptera: Salpingidae). Revista Chilena de Entomología, 46(3): 373-379.

Spilman, T.J. (1954) Generic names of the Salpingidae and their type species (Coleoptera). Journal of the Washington Academy of Science, 44: 85-94.

Villagrán,C. y Armesto,J.J. (2005) Fitogeografía histórica de la Cordillera de la Costa de Chile, pp. 99-116. En: Smith-Ramírez, C., Armesto, J.J. y Valdovinos, C. (eds.) Historia, Biodiversidad y Ecología de los bosques costeros de Chile. Editorial Universitaria, Santiago. 708 pp.

Villagrán, C. y Hinojosa, L.F. (2005) Esquema biogeográfico de Chile. Capítulo 33: pp. 551-577. En: Llorente Bousquets, J. y Morrone, J.J. (eds.) Regionalización Biogeográfica en Iberoámeríca y tópicos afines. Ediciones de la Universidad Nacional Autónoma de México, Jiménez Editores, México, 577 pp. 